\title{
Integrative Approach to the Management of Cardiometabolic Diseases
}

\author{
Gundu H. R. Rao ${ }^{1,2 *}$
}

'Emeritus Professor, Department of Laboratory Medicine and Pathology, Minneapolis, MN, USA ${ }^{2}$ Director Thrombosis Research, Lillehei Heart Institute, University of Minnesota, Minneapolis, MN, USA

\section{Article Info}

\section{Article Notes}

Received: May 01, 2018

Accepted: June 07, 2018

\section{*Correspondence:}

Dr. Gundu H. R. Rao, Emeritus Professor, Department of Laboratory Medicine and Pathology, Medical School, MN 55455, USA; Director Thrombosis Research Laboratory, Lillehei Heart Institute, University of Minnesota, Minneapolis, MN 55455, USA;

E-mail: gundura09@gmail.com

(c) 2018 Rao GHR. This article is distributed under the terms of the Creative Commons Attribution 4.0 International License.
When the editorial team of this journal invited me to write an article on integrative approach to healthcare, I was pleasantly surprised. Since this a very important approach and for some unknown reason, has remained obscured by the developments and applications of the Western Medical System, I decided to make an attempt to write my views on this topic. South Asians have a very high incidence of cardiometabolic diseases compared to any other ethnic group. Currently, China also has caught up with this rise in the incidence of excess weight, obesity and type- 2 diabetes. In view of this fact, we the members of South Asian community started a professional society, South Asian Society on Atherosclerosis and Thrombosis (SASAT) in1993, to address these issues. In our annual meetings in Bengaluru (2016), India, we organized a round table conference to discuss the needs and the importance of developing a unique health care platform, which includes the traditional medicine as a part of the affordable healthcare. With these ideas in mind, we started a platform called, Global Alliance of Traditional Health Systems (GATHS). In this overview, we will discuss the importance of traditional medicine in providing the much-needed complementary therapies. Since my area of interest is cardiometabolic disease, we will discuss this theme as apart of the novel ideas, in managing cardiometabolic disorders.

Cardiometabolic diseases such as hypertension, excess weight, obesity, metabolic syndrome, type- 2 diabetes, heart disease, and stroke, are increasing in their incidence worldwide, and in some countries, they have reached epidemic proportions. No country has been able to prevent the increase in the incidence of these chronic diseases. NCD Risk Factor Collaboration in their seminal article in the Lancet (April 2016) wrote, "if the post -2000 trends continue in the incidence and rise of diabetes, the probability of meeting the global target of halting the rise in the prevalence of diabetes by 2025 to 2020 level worldwide is less than one percent" ${ }^{\prime \prime}$. According to the Institute of Health Metrics and Evaluation (IHME), currently 2.1 billion people are either obese or overweight-a new, first of a kind analysis of trend data from 180 countries $^{2,3}$. Since 1980 , obesity has increased worldwide by two-fold and diabetes four-fold. Patients with diabetes have a two to three-fold higher risk for developing cardiovascular disease and cerebrovascular diseases. How did we reach this sorry state in spite of the tremendous improvements in health care worldwide? What other measures should we take to reduce or prevent these chronic metabolic diseases? In this review, we will try to address some of the issues and discuss some 
alternative or complementary approaches ${ }^{4-6}$.

If we are thinking of integrative approach for global heath, then we have to consider the concerns that the major Western countries have in adapting this approach and the efforts that they are making to include the integrative approach in their health care systems ${ }^{6}$. An integrative approach is a way of life, just like the holistic medicine is a way of life, compared to modern medicine, which is disease, focused. In recognition of the growing body of evidence about the importance of integrative approach to health, in 2009 Institute of Medicine (IOM), National Institutes of Health (NIH), USA, organized a summit on Integrative Medicine and Health of the Public. More than 600 health care providers participated in this summit. The IOM summit summary states, "The disease driven approach to care has resulted in spiraling costs as well as a fragmented health system that is reactive and episodic as well as inefficient and impersonal." In spite of such strongly worded summary statement, therapeutic aspects of traditional medicine in which herbal products or herbal preparations are used, have no place in the modern medicine, as US Food and Drug Administration (FDA) and regulatory agencies in Europe have banned sales of such products as therapeutic drugs.

Traditional Medicine practitioners of India and China have used herbal medicine and various phytochemicals for centuries and reaped the benefits of their medicinal properties. However, the regulatory agencies of US, UK and EU will not allow the sales of these drugs as therapeutics. In the USA, Public Health Services established a National Center for Complementary and Alternative Medicine (NCCAM) in 1991. Over 50 US academic institutions have complementary medicine programs funded by NCCAM. Dr Josephine Briggs the ex-director of this Institute says, "that the use of herbal medicine is not acceptable, as they do not meet the safety and efficacy criteria established by the US regulatory agencies (FDA). The name of this center has been changed to National Center for Complementary and Integrative Health (NCCIH). Now the question that rises in the minds of those who want to promote integrated health programs is, how do we accomplish this goal without doing very expensive often confusing and contradicting large clinical trials? Just consider what happened with the Fish Oil supplement studies. A recent issue of JAMA (April $18,2018)$ says. "Another Nail in the Coffin of the Fish Oil Supplements." After ten clinical trials with over 77, 000 patients the studies could not show any beneficial effect in cardiovascular outcomes. In view of such setbacks, supplement manufacturers do not want to take a risk and spend huge amounts of funds in the development of their products. We need to find a middle path that will ensure consumers the benefits of these health products with some supporting evidence-based data from randomized trials.
Let us take a look at some successful studies on supplements and phytochemicals to see if this approach is ideal for bringing the complementary medicine to the global market. David Sinclair, co-director of the Paul F Glenn Center for the Biology of Aging at Harvard Medical School says about NAD+, "It is one of the most important molecules for life to exist, and without it, you will be dead in 30 seconds." A randomized control trial found that people who took a daily supplement containing NAD+ precursors had a substantial, sustained increase in their NAD+ levels over a two-month period. A company called Elysium is selling a supplement called Basis that contains compounds that boost NAD+ levels. Leonard Guarente, Elysium's chief Scientist also directs the Glenn Center for Biology of Aging Research at MIT. Eight Nobel laureates are on the company's scientific advisory board and the company has partnered with such prestigious Academic institutions such as Harvard, MIT, Cambridge, and Oxford.

Inflammation seems to be the major initiator of many processes that leads to self-healing as well as pathogenesis of major metabolic disease, including, atherosclerosis, obesity, metabolic syndrome type-2 diabetes, heart disease and stroke. Inflammation seems to play a role even in acute events such as vulnerable plaque rupture, precipitation of some of the events associated with stroke, advance events in cancer and neuronal pathology. In spite of the great interest in the development of specialty drugs, for anti-inflammatory, anti-aging and anti-oxidant properties no specific drug is available to significantly modulate the levels of Nrf2, a nuclear transcription factor (erythroidderived 2). Recent studies however, have demonstrated that induction of Nrf2 and Ho-1 expression by Protandim (a mixture of five phytochemicals; Ashwagandha, Indian Bacopa, Indian Green Tea, China Milk Thistle and China Turmeric) is associated with a reduction in oxidative stress and fibrosis, preservation of the RV microcirculation and RV function ${ }^{8}$. Studies by Joe M McCord and associates on the effect of Protandim on various pathways have shown, significant modulation by Protandim not only of pathways involving antioxidant enzymes, but also those related to Colon Cancer, Cardiovascular disease and Alzheimer's disease $^{9,10,11}$. There seems to be a growing interest in the use of phytochemicals as modulators of multiple cellular pathways to prevent cancer and other chronic metabolic diseases. For instance, it has been shown that soy-derived glyceollins inhibit LNCaP prostate cancer cell growth similar to the effect observed with the soy isoflavone genistein, suggesting that a soy-base diet may be useful as a complementary dietary therapy for developing cancer preventive strategies $^{12}$.

Leading herbal product developer Himalayan Drugs, of India, has an anti-diabetic drug called, Diabecon, which contains ingredients from: Gymnema sylvestre, Pterocarpus 
marsupium, Glycyrrhiza glabra, Casearia esculenta, Syzgmium cumini, Asparagus racemosus, Boerhavia diffusa, Sphaeranthus indicus, Tinsopora cordifolia, Swertia chirata, Tribulus terrestris, Phyllanthus amarus, Gmelina arborea, Gossypium herbaceum, Berberis aristat, Aloe vera, Triphala, Commiphora wightii, shilajeet, Momordica charantia, Piper nigrum, Ocimum sanctum, Abuilton indicum, Curcuma longa, Rumex maritimus. Whereas, another product Diasulin contains; Cassia auriculata, Coccinia indica, Curcuma longa, Emblica officianlis, Syzugium cumini, Trigonella foenum graecum, Azadrichta indica, Ficus racemosa, Aegle marmelos, Cinnamomum tamala. Some Ayurvedic products have Gurmar (Gymnema sylvestre) Karela (Momordica charantia) Pushkarmool (Inula recemosa), Jamun gutli (Syzygium cumini) Neem (Azardochta indica) Methika (Trigonella foenum gracecum) Guduchhi (Tinospora cordifolia) ${ }^{13-15}$. Problem with such formulations with multiple phytochemicals is lack of specificity lack of therapeutic potency of individual ingredients and the problems associated with standardization and evaluation of bioavailability, safety and efficacy of these formulations.

A search of literature on this topic (anti-diabetic, antiobesity herbal drugs) reveals many herbal products, their extracts, active ingredients and supplements that could be used for managing diabetes and excess weight. However, no guidelines exist as to how to go about developing a reasonable evidence-based support for the claims made by these preparations. If one establishes a clear goal, develops appropriate screening methods with specific end points, then one can develop, reasonable evidence to support their claims as well as establish some degree of standardization of their products. Readers are urged to refer to original reviews and books to get additional information on this topic $^{16}$. Having said that, we would like to give couple of examples to show how one can develop validation studies to establish the efficacy of such preparations in a small number of subjects in the shortest possible time and with a limited budget. First of all, we would like to introduce the use of new glucose sensor developed by Abbott healthcare (FreeStyle Libre-ACGM System) for continuous monitoring glucose to demonstrate the beneficial effects of newly developed indigenous formulations on postprandial glucose peaks (www.freestylelibre.us). Secondly, we would like to introduce an integrated non-invasive diagnostic platform, to assess the effectiveness of drug formulations on collective risks of cardiometabolic diseases.

What these studies demonstrate, is that by using observation-based studies one can demonstrate the usefulness of supplements and phytochemicals. We at the Karnataka Institute of Endocrinology and Research, Bengaluru, have demonstrated in our earlier studies the beneficial effect of mulberry green tea in lowering the postprandial glucose (Personal Observations) levels using the classical analytical methods. Now that Abbott has developed a glucose sensor, which monitors interstitial glucose every fifteen minutes, we can do such studies and get the needed information (real time data) on the beneficial effects of anti-glycemic drugs by following minimal number of patients. This methodology will be very useful to screen drugs that can modulate the post meal glucose peaks. As seen in the above illustration, it would be useful tool for the education of diabetic subjects in terms of risk assessment, risk prediction and risk reduction. We at the Department of Biochemistry, All India Institute of Medical Sciences, Patna, are studying the usefulness or otherwise of this approach for screening anti-glycemic drugs, phytochemicals, herbal products and dietary supplements, under the leadership of Professor Sadhana Sharma, Head of the Department of Biochemistry.

We are also using such emerging technologies for monitoring and computing cardiometabolic risks (CMR). At the Institute for Preventive Cardiology (IPC Heartcare, Mumbai), under the leadership of Dr. Pratiksha Gandhi, the Director IPC healthcare, we are using TM Oxi system to monitor cardiometabolic risks ((LD-technologies, Miami, Florida: www.ldteck.com). The non-invasive platform uses oximeter, blood pressure monitors and a galvanic skin response monitor to obtain the needed data. A typical print out from this system is presented below. The data on parameters like neuronal activities, lipid markers, blood pressure, IGT markers are algorithm derived and results are expressed as Cardiometabolic risk score (CMRs) and Autonomous Nervous Risk Score (ANRs). For additional details about the use of this technology please refer to the following publications ${ }^{17-19}$.

Using such simple methods or devices that are dedicated (Periscope, Genesis Medical Systems Hyderabad, India, CVprofiler System, Hypertension Diagnostics, Minnesota) one can follow, heart rate variability, endothelial dysfunction and other risks for CMDs. If one can develop a method for monitoring reliable biomarkers, then it is easy to demonstrate the beneficial effects or otherwise of molecules, compounds, supplements and dietary interventions. We as a part of our bilateral collaboration at the IPC Heartcare, Mumbai, India (www.ipcheartcenter. com), are evaluating, green tea of mulberry leaves as well as green tea from regular tealeaves as supplements to manage postprandial hyperglycemia. A major green tea polyphenol, epigallocatechin-3-gallate, inhibits obesity, metabolic syndrome and fatty liver in high-fat-fed mice model. In addition to the tea mentioned above, we also are evaluating D-ribose, L-arginine, Curcumin, Carnitine, Resveratrol, Anthocyanin-rich blue-berry extract, citrus-derived flavonoid Narangenin, grape seed extract Procyanidon, green tea extract Tegreen, mulberry leaf extract 1-deoxynojirimcin (DNJ), water soluble extract of 


\section{Glucose Pattern Insights report (continued)}

The Glucose Pattern Insights report includes an key parameters for glucose control in the form of red, yellow, green stop lights to assess the risk level.

\section{Parameters:}

Likelihood of Low Glucose is the probability that low-glucose values have exceeded an allowable, user defined threshold.

Median Glucose is an indication of when the median glucose has exceeded a user defined goal.

Median glucose is strongly correlated to A1c.

Variability Below Median is a measure of the spread of glucose data below the median. It is calculated as the difference between the 50th and 10th percentile glucose readings for the time period.

Risk level is assessed for 5 time periods (there are 2 periods between Bedtime and Breakfast) in the "typical" day.

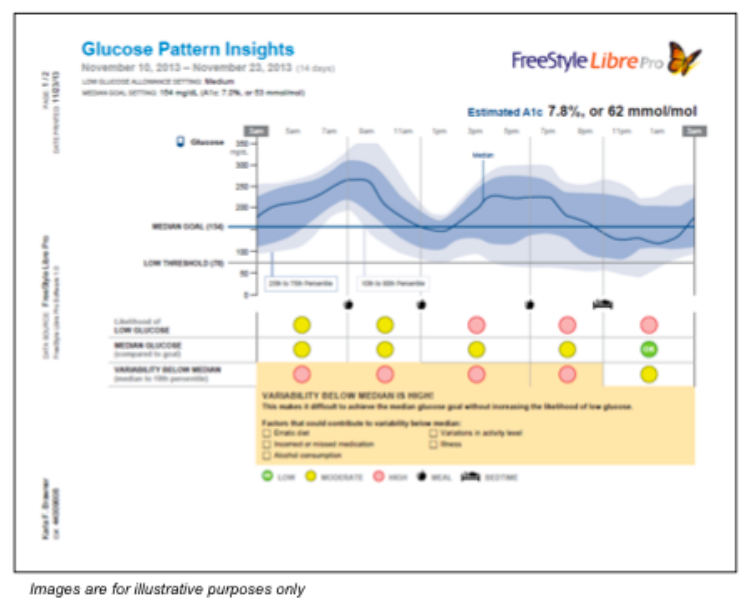

Figure 1; Figure shows a standard interstitial glucose profile for a twelve-hour period. The spread of glucose data shows below the median values, as well as above the median (dark blue line). The glucose measurement is recorded every fifteen minutes. According to the manufacturers, the median glucose values are strongly correlated with the HBA 1c. Risk level is assessed five times between bedtime and breakfast in a typical day.

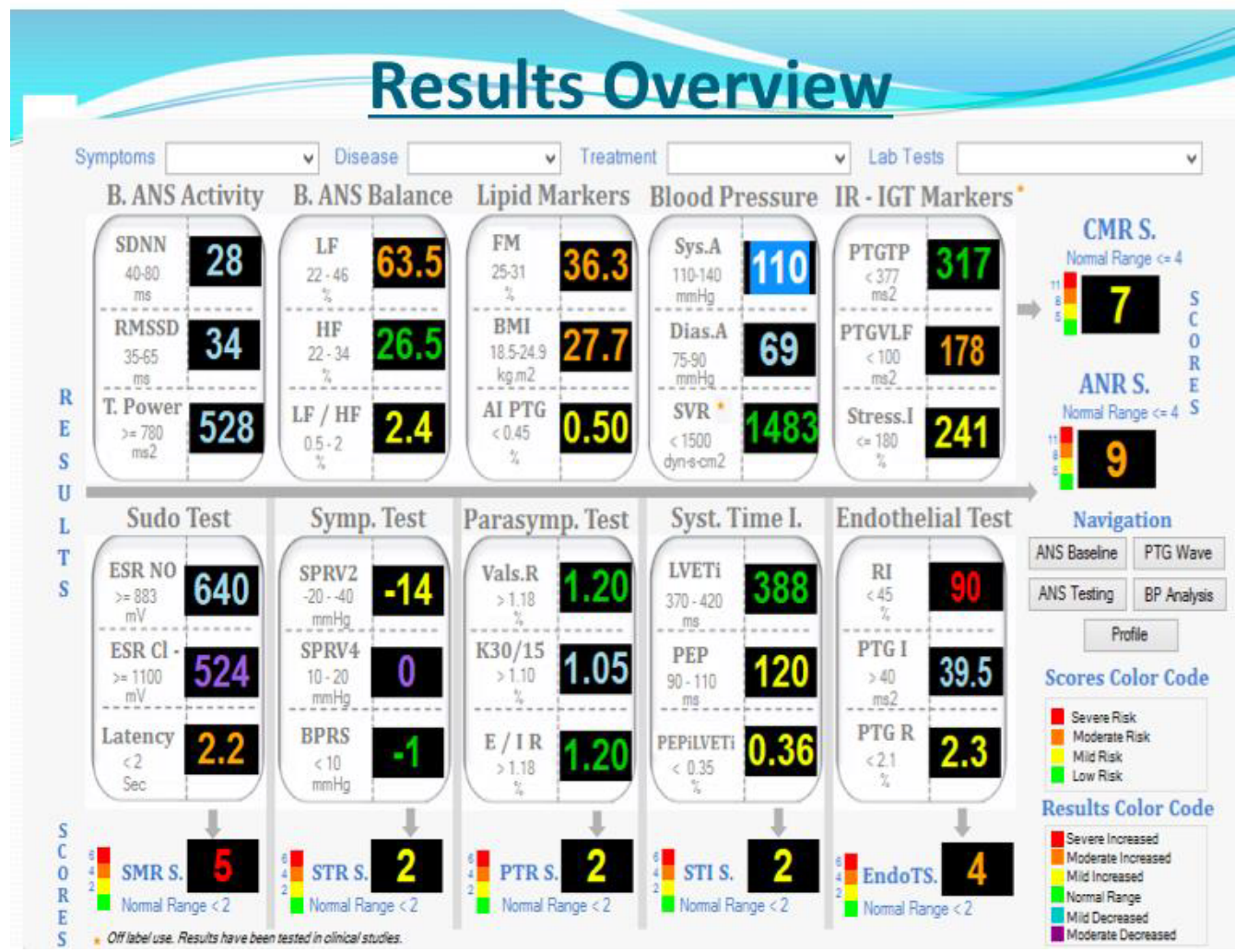

Figure 2: Figure shows results of a typical study using the Tm-Oxi and SudoPath systems. There are ten different tests reported. Each test result is color coded to indicate the level of risk from green to red, green being safe and red being "severe risk". Results also are presented with separate risk scores for various risk clusters. For instance, CMRs refers to cardiometabolic risk score. 
cinnamon and coffee (Green Coffee), and hibiscus tea. Based on the results of our studies, we plan to screen a variety of phytochemicals known to lower glycemic load as well as develop a "poly-pill" with indigenous phyto-chemicals for the management of postprandial hyperglycemia. We will also develop nutritional supplements as well as diet drinks for the diabetic population using some of these novel ideas. Since we use TM- Oxi system (LD-Technologies, Miami Florida) to monitor risk profiles of patients, we can evaluate various indigenous therapies for their effectiveness or otherwise, in managing the observed risks of MS, T2D and CVDs, using this non-invasive diagnostic platform.

Now that we have discussed few novel ideas to make a case for the use of complementary medicine, we need to discuss as to what is preventing us from using life style change, diet and personalized healthcare in integrated preventive strategies. Risks for chronic diseases, develops over a long period of time and cannot be detected without a visit to the clinic and appropriate laboratory tests. By and large people, in majority of the countries people visit clinic only when they are sick. In most of the countries, there is very little incentive or funds for prevention programs. In our opinion efforts for prevention of the metabolic disease should start at the earliest and continue for the entire life. We should integrate adolescent NCD prevention and treatment into basic primary health care services and packages, in particular Reproductive, Maternal, Newborn, and Child Heath (RMNCH) platforms, to screen for those in need of NCD treatment. We should promote healthy behaviors across the life cycle, beginning with maternal health and the pre-natal period and extending throughout childhood and adolescence and into adulthood.

If we look at the commercial success of herbal products, Chyavanaprasa an Ayurvedic formulation of amla, ashwagandha, pippali, cardammon, nutmeg and cinnamon has a market share of over 100 million. The manufacturers claim all kinds of health benefits citing its ancient origin from Vedic times. Similarly, Baba Ramadev an exponent of Yoga and a Media savvy individual, using the name of Patanjali, the original author on Yogic principles (Yoga Sutra by Patanjali) has developed a variety of herbal preparations under the brand name of "Patanjali" and very successfully marketed them all over India. If we look at the success of Elysium, its scientific board has eight noble prizewinners and has academic partnership with Harvard, MIT, Cambridge and Oxford. LifeVantage a wellness company makers of Protandim, claims that they are a science based visionary herbal product Development Company. Going over just a few success stories, it is evident that there are a variety of strategies for marketing potent phytochemicals and their combinations as supplements for complementary therapy of oxidative stress, inflammation, aging and chronic metabolic diseases. Development of supplements with evidence-based support for their efficacy and safety will enable this industry to play a very important role in affordable healthcare by providing welltested complimentary therapeutics.

\section{References}

1. NCD Risk Factor Collaboration (NCD-RisC): Trends in adult bodymass index in 200 countries from 1975 to 2014; a pooled analysis of 1968 population-based measurement studies with 19.2 million participants. The Lancet 387:1377-1396, 2016.

2. McKee M, Haines A, Ebrahim S, et al. Towards a comprehensive global approach to prevention and control of NCDs Globalization and Health. $2014 ; 10: 74$

3. NCD Alliance. NCDs across the SDGs: A call for an integrated approach, NCD alliance, May 2017. https://ncdalliance.org/sites/default/files/ resource_files /NCDs_Across_SDGs_English_May2017.pdf

4. NCD Risk Factor Collaboration (NCD-RisC) Worldwide trends in body mass index, underweight, and obesity from 1975to 2016. A pooled analysis of 2416 population-based measurement studies from 128.9 million children adolescents and adults. The Lancet. 2017; 1-16.

5. Rao GHR. Prevention and reversal of cardiometabolic diseases. J Clin Prevent Cardiol. 2018; 7(1): 22-28.

6. Rao GHR:. Integrative approach to health: Challenges and opportunities. J Ayurveda Integr Med. 2015; 6(3): 215-219.

7. Rao GHR. Opportunities and Challenges in Ayurveda: Global Perspective. Altern \& Integr Med. 2017; 6: 239. doi10.4172/23275162.1000239.

8. Rao GHR. Bharathi M: Mother and child: First step for prevention of cardiometabolic disease. The Cardiol (Photon Journal) Photon. 2016; 109: 179-186.

9. Boggard HJ, Natarajan R, Henderson R, et al. Chronic pulmonary artery pressure elevation is insufficient to explain right heart failure. Circ. 2009; 1201951-60.

10. Hybertson BM, Gao B, Bose SK, et al. Oxidative stress in health and disease: The therapeutic potential of Nrf2 activation. Mol Aspects of Med. 2011; 32: 234-46.

11. Joe M McCord, Fridovich I. The biology and pathology of oxygen radicals. Ann Int Med. 1978; 89(1): 122-27.

12. Redox Pioneer: Professor Joe McCord. Antioxid. Redox Signal. 2014; 20 (!): 183-188.

13. Mahmoud AM, Yang W, Bosland MC. Soy Isoflavones and prostate cancer A review of molecular mechanisms. The J Steroid Biochem and Mol Biol. 2014; 140: 116-132.

14. Maarek AA, Gandhi PG, Rao GHR. Identifying autonomic neuropathy and endothelial dysfunction in type-2 diabetic patients. EC .Neuropathy. 2015; 2: 63-78,

15. Modak M, Dixit P, Londhe J, et al. Indian herbs and Herbal Drugs used for the Treatment of Diabetes. J Clin. Biochem Nutr. 2007; 40 (3): 163 173.

16. Sharma RR. Preliminary phytochemical screening of some indigenous medicinal plant leaves extract in regulation of antidiabetic activity. Sci Res Report. 2012; 2 (3): 307-310.

17. Integrative Approaches for Health ((Patwardhan B, Mutalki G, Tillu G. Eds), Elsevier Inc. ISBN. 2015; 978-0-12-801282-6.

18. Rao GHR. Non-traditional approaches to diagnosis and management of diabetes mellitus: Point of view. J Diabetes Metab. 2015; 6: 489. Doi:10.4172/2155-6156.1000489. 
19. Gandhi PG, Rao GHR. The spectral analysis of photoplethysmography to evaluate an independent cardiovascular risk factor. Int Gen Med. 2014; $7: 539-547$.
20. Gandhi PG, Rao GHR. Detection of neuropathy using a SudoMotor test in type-2 diabetes. Degenerative Neurological and neuromuscular Disease. 2015; 5: 1-7. 